\title{
Additive preventive effect of influenza and pneumococcal vaccines in elderly persons
}

\author{
B. Christenson*, J. Hedlund", P. Lundbergh*, Å. Örtqvist*,\#
}

Additive preventive effect of influenza and pneumococcal vaccines in elderly persons. B. Christenson, J. Hedlund, P. Lundbergh, A. Örtqvist. (C) ERS Journals Ltd 2004. ABSTRACT: In 1999, all individuals $\geqslant 65 \mathrm{yrs}$ of age $(\mathrm{n}=258,754)$ in Stockholm County, Sweden, were offered influenza and pneumococcal vaccination in a prospective study on the effectiveness of these vaccines in reducing the need for hospital treatment and death due to influenza, pneumonia and invasive pneumococcal disease (IPD). Data on hospitalisation and mortality during $1 \mathrm{yr}$ were obtained from the administrative database in Stockholm County Council.

Vaccination was performed in $124,702(48 \%)$ subjects; 72,107 had both vaccines, 29,346 only had the influenza vaccine and 23,249 only had the pneumococcal vaccine. Compared with the unvaccinated cohort, a lower incidence of hospitalisation for all endpoint diagnoses was seen in vaccinated persons.

An additive effectiveness of vaccination was seen when both vaccines were given, with a reduction of hospital admissions for influenza (37\%), pneumonia (29\%) and IPD $(44 \%)$. In-hospital mortality for pneumonia was significantly lower in those who received both vaccines, than in unvaccinated persons.

To conclude, vaccination with influenza and pneumococcal vaccines together was effective in reducing the need for hospital admission for influenza and pneumonia. There was a strong indication that pneumococcal vaccination alone, was effective not only in the prevention of invasive pneumococcal disease, but also of pneumonia overall, although to a low degree.

Eur Respir J 2004; 23: 363-368.
*Dept of Communicable Disease Control and Prevention, Stockholm County, and ${ }^{\#}$ Dept of Infectious Diseases, Karolinska Institutet, Karolinska Hospital, S-171 76 Stockholm, Sweden.

Correspondence: A. Örtqvist, Dept of Communicable Diseases and Prevention, Norrbacka, Karolinska Hospital, SE-171 76 Stockholm, Sweden.

Fax: 468315767

E-mail: ake.ortqvist@sme.sll.se

Keywords: Elderly

influenza vaccine

pneumococcal vaccine

Received: June 52003

Accepted after revision: September 162003

This study was supported financially by Stockholm City Council, The Swedish Heart-Lung Foundation, and Karolinska Institutet.
Influenza and pneumococcal diseases are common causes of hospitalisation and excess death in elderly persons. The risk of complications, hospitalisation and death from influenza and pneumonia is higher among persons aged $\geqslant 65 \mathrm{yrs}$ than in younger persons [1-3]. The use of influenza and pneumococcal vaccines has been limited in many European countries [4], despite recommendations of annual vaccination of the elderly to prevent hospitalisation and death $[5,6]$. This has been partly attributable to uncertainty regarding the benefit of these vaccines, especially concerning the benefit of pneumococcal vaccination.

Previous prospective randomised studies of the 23-valent pneumococcal vaccine in elderly people in preventing hospitalisation and death due to pneumococcal pneumonia have been inconclusive [7-10]. However, these studies all lacked in power. Furthermore, one trial from France did indicate that the pneumococcal vaccine was efficacious against pneumonia in a nursing home setting [10]. More recently, a retrospective cohort study demonstrated that pneumococcal vaccination reduced hospitalisation and death due to pneumonia in elderly people with chronic lung disease [11].

There has been less doubt about the evidence of the protective effect of pneumococcal vaccine in preventing invasive pneumococcal disease (IPD). In case-control and indirect cohort studies, a $60-80 \%$ effectiveness of pneumococcal vaccination in preventing IPD has been shown [12-15].

Concerning influenza immunisation in elderly people, several studies have indicated the benefit of annual influenza vaccination, especially during epidemic years $[3,16]$, but the efficacy of the vaccine in reducing more severe, influenzarelated diseases requiring hospital admissions has been shown only in case-control and retrospective cohort studies [17-21].

In Sweden, the vaccination rate of influenza and pneumococcal vaccines has been low, probably partly because that vaccination has not been reimbursed. There is no general recommendation by age for pneumococcal vaccination, but since 1994 the vaccine may be considered in individuals of $\geqslant 65$ yrs according to the Swedish National Board of Health and Welfare. An annual influenza vaccination of this age group has been recommended only since 1997 .

This study is part of a 3-yr prospective intervention study on the impact of influenza and pneumococcal vaccinations in reducing hospitalisation and death among the elderly population of Stockholm County. All individuals in Stockholm County aged $\geqslant 65$ yrs were invited to participate in a vaccination campaign against influenza and pneumococcal infections during three consecutive years, 1998-2000. Two reports from the first year of the vaccination campaign have been published $[22,23]$. In these studies, it was found that the incidence of hospital admissions were lower in the vaccinated than in the unvaccinated cohort for influenza, pneumonia and IPD. Vaccinated persons also had a lower mortality due to pneumonia, chronic obstructive pulmonary disease and cardiac failure.

This article is an analysis of the second of the 3-yr study. Based on the large accumulated patient population, the effectiveness of each the two vaccines was addressed separately, as well as their additive effect. 


\section{Material and methods}

\section{Study population}

All individuals aged $\geqslant 65 \mathrm{yrs}$ were invited by a personal letter to receive, at reduced cost, influenza and pneumococcal vaccines during an 8-week period from the end of September to the middle of November for each of the years 1998-2000. The vaccination campaign was also advertised in the daily papers and on posters at general practitioners' offices and at pharmacies.

In 1998, the total study population was 259,627, and of these 77,177 received both vaccines, 23,224 received only the influenza vaccine and 841 only the pneumococcal vaccine [22, 23]. In 1999 (the main cohort for the current analysis), the study population was largely the same as in 1998. However, the 1999 cohort also included persons who that year became $65 \mathrm{yrs}$ of age, but excluded those who had died during 1998. The resulting number of persons of $\geqslant 65 \mathrm{yrs}$ of age was therefore 258,747, of which 134,045 did not accept the offer of vaccination. Of those in the vaccinated group, 72,107 received both vaccines, 29,346 received only the influenza vaccine and 292 only the pneumococcal vaccine. In addition, 22,957 individuals who had received the pneumococcal vaccination in 1998, and who did not accept the offer of influenza vaccination in 1999, were included in the analysis.

The vaccines used were the 23 -valent pneumococcal polysaccharide vaccine (Pneumovax from Pasteur-Merieux MSD, Pennsylvania, PA, USA; Pnu-Imune from Wyeth-Lederle, New York, NY, USA; or Pneumokockvaccin from SBL Vaccin, Stockholm, Sweden) and a trivalent split-virion influenza vaccine (Begrivac; Chiron Behring $\mathrm{GmbH} \& \mathrm{Co}$, Marburg, Germany, 1999/2000) containing $15 \mu \mathrm{g}$ of $\mathrm{A} /$ Beijing/262/95 (H1N1), A/Sydney/5/97 (H3N2) and B/Harbin/ 7/94 (B/Beijing/184/93-like strain).

General practitioners performed most of the vaccinations. At vaccination, the physician noted each individual's name and personal identification code (unique for each Swedish citizen), and whether one or both vaccines were given. These data were then entered in a database at the Department of Communicable Disease Control and Prevention. For analyses, the vaccination data were matched with discharge diagnoses according to the International Classification of Diseases, tenth revision (ICD-10-CM), together with mortality data for all individuals aged $\geqslant 65 \mathrm{yrs}$ hospitalised in Stockholm County from December 1999 through November 2000.

A questionnaire concerning underlying health and demographic data was sent to a random sample of 10,000 individuals aged $\geqslant 65 \mathrm{yrs}$ in Stockholm County between December 2000 and January 2001 with the aim to characterise possible confounders in the comparison of the vaccinated and unvaccinated cohorts. The results of this study have been published elsewhere [24].

\section{End-points}

The primary end-points were incidence of hospital admissions, number of days in hospital, and in-hospital deaths due to influenza (ICD-10: J10.0, J10.1, J10.8, J11.0 and J11.8), pneumonia (ICD-10: J12-18, J69.0 and A48.1) and IPD (ICD-10: A40.3 and G00.1) in the vaccinated versus the unvaccinated cohort. An end-point diagnosis was accepted irrespective of whether it was on the first, second or third of the discharge diagnoses. However, only one end-point diagnosis, the first to appear, per episode of hospital treatment was included in the analysis.

The vaccinated cohort included individuals who received either influenza or pneumococcal vaccine or both vaccines. Comparisons with the unvaccinated cohort were performed against the total vaccinated cohort (receiving one or both vaccines), as well as against those who received influenza or pneumococcal vaccine only, and against those who had received both vaccines. The incidence of hospital admissions and in-hospital deaths were compared for the end-point diagnoses during the 12-month study period, December 1999 through November 2000. In addition, the cohorts were compared during the influenza season when influenza virus was circulating in the community, December 1999 through May 2000, and the noninfluenza season, June 2000 through November 2000. During the influenza season, there was a medium-sized, influenza A (Sydney H3N2) epidemic in Stockholm County.

\section{Statistical methods}

The statistical differences between vaccinated and unvaccinated individuals were evaluated, using the confidence interval for a proportion and the Chi-squared test for categorical variables. The reduction in mortality in vaccinated people was calculated from the results of the logistic regression analysis. The adjusted odds ratio (OR) was used as an estimate of the relative risk. Thus, the reduction in mortality was calculated as $(1-\mathrm{OR}) \times 100 \%$.

\section{Results}

In total, 124,702 individuals ( $48 \%$ of the target population) were vaccinated with one or both vaccines. Of these, 101,745 individuals $(39 \%)$ were vaccinated during the 8 -week vaccination campaign in 1999 and 22,957 had received pneumococcal vaccination during the vaccination campaign in 1998, but no influenza vaccination in 1999. Both vaccines were given to 72,107 individuals, the influenza vaccine alone to 29,346 , and the pneumococcal vaccine alone to 23,249 persons. Of the vaccinated individuals, 59\% were females, a distribution similar to that of all persons in these age groups in Stockholm County ( $60 \%$ females). Table 1 shows the distribution of the

Table 1.-Proportions of vaccinated individuals and vaccines given in different age groups

\begin{tabular}{|c|c|c|c|c|c|}
\hline \multirow[t]{2}{*}{ Age yrs } & \multirow[t]{2}{*}{ Individuals $\mathrm{n}$} & \multirow[t]{2}{*}{ Vaccinated individuals $\#$} & \multicolumn{3}{|c|}{ Vaccine given } \\
\hline & & & Influenza $\%$ & Pneumococcal $^{+} \%$ & Both $^{\S} \%$ \\
\hline $65-74$ & 124428 & 46 & 10 & 8 & 28 \\
\hline $75-84$ & 99118 & 52 & 12 & 9 & 30 \\
\hline$\geqslant 85$ & 35201 & 46 & 13 & 10 & 23 \\
\hline Total & 258747 & 48 & 11 & 9 & 28 \\
\hline
\end{tabular}

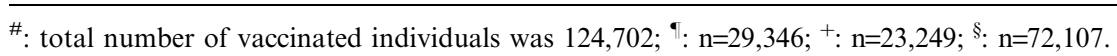


Table 2. - Hospital admissions for influenza (with and without influenza pneumonia), pneumonia, and invasive pneumococcal disease (IPD) per 100,000 individuals in total vaccinated $(n=124,702)$ and unvaccinated $(n=134,045)$ cohorts in different age groups

\begin{tabular}{|c|c|c|c|c|c|c|}
\hline \multirow{2}{*}{$\frac{\text { Diagnosis }}{\text { Vaccinated }}$} & \multicolumn{2}{|c|}{ Influenza } & \multicolumn{2}{|c|}{ Pneumonia } & \multicolumn{2}{|c|}{ IPD } \\
\hline & Yes & No & Yes & No & Yes & No \\
\hline Subjects $65-74$ yrs n & 80 & 82 & 865 & 1108 & 7 & 27 \\
\hline $\begin{array}{l}\text { OR }(95 \% \mathrm{CI}) \\
\text { p-value }\end{array}$ & \multicolumn{2}{|c|}{$0.97(0.66-1.46)$} & \multicolumn{2}{|c|}{$0.78(0.69-0.87)$} & \multicolumn{2}{|c|}{$0.26(0.086-0.78)$} \\
\hline Subjects $75-84$ yrs $n$ & 162 & 282 & 2057 & 2726 & 27 & 46 \\
\hline $\begin{array}{l}\text { OR }(95 \% \mathrm{CI}) \\
\text { p-value }\end{array}$ & \multicolumn{2}{|c|}{$<0.0001$} & \multicolumn{2}{|c|}{$<0.0001$} & $0.59(0.30-1.18)$ & $<0.12$ \\
\hline $\begin{array}{l}\text { Subjects }>85 \text { yrs n } \\
\text { OR }(95 \% \mathrm{CI}) \\
\text { p-value }\end{array}$ & \multicolumn{2}{|c|}{$\begin{array}{c}0.60(0.40-0.86) \\
<0.007\end{array}$} & $\begin{array}{r}4487 \\
0\end{array}$ & $3)^{5288}$ & 6 & 16 \\
\hline $\begin{array}{l}\text { Subjects }>65 \text { yrs n } \\
\text { OR }(95 \% \text { CI }) \\
\text { p-value }\end{array}$ & $\begin{array}{r}136 \\
0\end{array}$ & 201 & $\begin{array}{r}1821 \\
0\end{array}$ & 4) & ${ }^{15}$ & 32 \\
\hline
\end{tabular}

OR: odds ratio; CI: confidence interval. All p-value comparisons are against the unvaccinated cohort.

study population by age group, the proportion of vaccinated persons and the vaccine given.

Comparison of the rates of hospitalisation in different age groups between all vaccinated individuals $(124,702)$ and those unvaccinated $(134,045)$ showed that the incidence of hospital admissions for all diagnoses was lower among vaccinated individuals in most, but not all, age groups (table 2). For influenza, no effect was seen in the youngest age group (65-74 yrs of age), while a significant effect for prevention against IPD was seen only in that age group.

Compared with the unvaccinated group, the risk for being admitted to hospital for influenza, with or without pneumonia, was significantly lower for those who had received both vaccines, OR 0.63 (95\% confidence interval (CI) $0.50-0.81$; table 3 ). The same tendency, although not quite significant, was seen in those who received only the influenza vaccine, or only the pneumococcal vaccine. Similarly, there was a tendency for the two vaccines given separately to reduce admissions for pneumonia, compared with the unvaccinated cohort, while the two vaccines given together were clearly additive and resulted in a significantly lower risk, OR 0.71 (95\% CI $0.65-0.75)$. The incidence of admissions due to IPD was $44-73 \%$ lower in those who received one or both vaccines, compared with no vaccination, but the number of cases was too low to obtain a statistical significant difference (table 3).

An additive effect of the two vaccines was also seen for inhospital mortality due to pneumonia with a $35 \%$ reduction
(95\% CI 22-46) compared with the unvaccinated group (table 4). A tendency to an additive effect of the two vaccines was also seen for influenza-related mortality, but the number of fatal cases was low resulting in a wide CI (OR $0.29,95 \% \mathrm{CI}$ $0.06-1.31)$. The overall mortality was $\sim 40 \%$ lower in the vaccinated cohort $(n=124,702)$, compared with the unvaccinated cohort $(n=134,045)$, for all age groups (table 5). The number of deaths per 1,000 individuals of $\geqslant 65$ yrs was 39 in the vaccinated versus 61 in the unvaccinated cohort $(\mathrm{p}<0.0001)$.

The duration of hospital treatment for influenza and pneumonia was significantly shorter in the vaccinated cohort compared with the unvaccinated cohort (data not shown). In those who had received both vaccines, the mean duration of hospital stay in vaccinated versus nonvaccinated was 5.2 versus 7.5 days $(\mathrm{p}<0.005)$ for influenza and 9.9 versus 11.3 days $(\mathrm{p}<0.0004)$ for pneumonia. Fewer hospitalisations and shorter duration of hospital treatment in the vaccinated cohort led to a reduction of the total number of days in hospital; $56 \%$ for influenza, $38 \%$ for pneumonia and $48 \%$ for IPD.

During the influenza season, December 1999 through May 2000, a significant reduction in hospital admissions was found for influenza, pneumonia and IPD in persons who had received both vaccines compared with the unvaccinated group (table 6). During the noninfluenza season, June through November 2000, there was still a significantly lower risk for pneumonia in the vaccinated group compared with the

Table 3. - Hospital admissions for influenza (with and without influenza pneumonia), pneumonia and invasive pneumococcal disease (IPD), per 100,000 individuals

\begin{tabular}{lccc}
\hline Diagnosis & \multicolumn{2}{c}{ Vaccine given } \\
\cline { 2 - 4 } & Influenza & Pneumococcal & Both \\
\hline Subjects n & 29346 & 23249 & 72107 \\
Influenza & 150 & 142 & 128 \\
OR (95\% CI) & $0.74(0.54-1.03)$ & $0.70(0.49-1.03)$ & $0.63(0.5-0.81)$ \\
p-value & $<0.1$ & $<0.1$ & $<0.001$ \\
Pneumonia & 2140 & 2082 & 1607 \\
OR (95\% CI) & $0.94(0.86-1.02)$ & $0.91(0.82-1.00)$ & $0.71(0.65-0.75)$ \\
p-value & $<0.2$ & $<0.06$ & $<0.0001$ \\
IPD & 14 & 9 & 18 \\
OR (95\% CI) & $0.42(0.15-1.21)$ & $0.27(0.06-1.14)$ & $0.56(0.30-1.05)$ \\
p-value & $<0.1$ & $<0.06$ & $<0.06$
\end{tabular}

OR: odds ratio; CI: confidence interval. Data taken from subjects after receiving separate influenza or pneumococcal vaccines, or both, compared with no vaccine, between December 1, 1999 and November 30, 2000. All p-value comparisons are against the unvaccinated cohort. 
Table 4.-In-hospital mortality per 100,000 individuals after receiving separate influenza or pneumococcal vaccines, or both, compared with no vaccine, between December 1, 1999 and November 30, 2000

\begin{tabular}{lccc}
\hline Diagnosis & \multicolumn{3}{c}{ Vaccine given } \\
\cline { 2 - 4 } & Influenza & Pneumococcal & Both \\
\hline Subjects n & 29346 & 23249 & 72107 \\
Influenza & 6.8 & 4.3 & 2.8 \\
OR (95\% CI) & $0.70(0.15-3.21)$ & $0.44(0.06-3.53)$ & $0.29(0.06-1.31)$ \\
p-value & $<0.64$ & $<0.42$ & $<0.09$ \\
Pneumonia & 299.9 & 318.3 & 223.3 \\
OR (95\% CI) & $0.88(0.69-1.11)$ & $0.93(0.72-1.19)$ & $0.65(0.54-0.78)$ \\
p-value & $<0.24$ & $<0.56$ & $<0.0001$ \\
\hline
\end{tabular}

OR: odds ratio; CI: confidence interval. ${ }^{\#}$ : with and without influenza pneumonia. All p-value comparisons are against the unvaccinated cohort.

Table 5. - Deaths from all causes according to age and vaccination status between December 1, 1999 and November 30,2000

\begin{tabular}{|c|c|c|c|c|c|c|c|c|}
\hline \multirow[b]{3}{*}{ Vaccinated $^{\#}$} & \multicolumn{8}{|c|}{ Age yrs } \\
\hline & \multicolumn{2}{|c|}{$65-74$} & \multicolumn{2}{|c|}{$75-84$} & \multicolumn{2}{|c|}{$\geqslant 85$} & \multicolumn{2}{|c|}{$\geqslant 65$} \\
\hline & Yes & No & Yes & No & Yes & No & Yes & No \\
\hline Individuals $\mathrm{n}$ & 57377 & 67051 & 51280 & 47838 & 16045 & 19156 & 124702 & 134045 \\
\hline Deaths per 1000 & 14.6 & 23.5 & 41.0 & 64.1 & 121.8 & 185.9 & 39.2 & 61.2 \\
\hline OR $(95 \% \mathrm{CI})$ & \multicolumn{2}{|c|}{$0.61(0.56-0.67)$} & \multicolumn{2}{|c|}{$0.62(0.59-0.66)$} & \multicolumn{2}{|c|}{$0.61(0.57-0.65)$} & \multicolumn{2}{|c|}{$0.63(0.60-0.65)$} \\
\hline p-value & \multicolumn{2}{|c|}{$<0.00001$} & \multicolumn{2}{|c|}{$<0.00001$} & \multicolumn{2}{|c|}{$<0.0001$} & \multicolumn{2}{|c|}{$<0.00001$} \\
\hline
\end{tabular}

OR: odds ratio; CI: confidence interval. ${ }^{\#}$ : vaccinated with one or both vaccines.

Table 6. - Hospital admissions per 100,000 individuals per year for influenza (with and without pneumonia), pneumonia, and invasive pneumococcal disease (IPD)

\begin{tabular}{|c|c|c|c|c|c|c|}
\hline \multirow{2}{*}{$\frac{\text { Diagnosis }}{\text { Vaccinated }}$} & \multicolumn{2}{|c|}{ Influenza } & \multicolumn{2}{|c|}{ Pneumonia } & \multicolumn{2}{|c|}{ IPD } \\
\hline & Yes & No & Yes & No & Yes & No \\
\hline Influenza season & 247 & 391 & 1784 & 2751 & 13 & 49 \\
\hline $\begin{array}{l}\text { OR }(95 \% \mathrm{CI}) \\
\text { p-value }\end{array}$ & \multicolumn{2}{|c|}{$\begin{array}{c}0.63(0.53-0.75) \\
<0.0001\end{array}$} & \multicolumn{2}{|c|}{$0.64(0.60-0.69)$} & \multicolumn{2}{|c|}{$0.28(0.14-0.55)$} \\
\hline Noninfluenza season & 8 & 12 & 1431 & 1814 & 19 & 15 \\
\hline $\begin{array}{l}\text { OR }(95 \% \text { CI }) \\
\text { p-value }\end{array}$ & \multicolumn{2}{|c|}{$0.47(0.15-1.42)$} & & $0.79(0.73-0.85)$ & & \\
\hline
\end{tabular}

Data taken from individuals who had received both vaccines $(n=72,107)$, compared with unvaccinated persons $(n=134,045)$ during the influenza season (December 1, 1999 to May 31, 2000), and the noninfluenza season (June 1, 2000 to November 30, 2000). OR: odds ratio; CI: confidence interval. All p-value comparisons are against the unvaccinated cohort.

unvaccinated group, while there was no difference in the rate of hospitalisation for influenza or IPD.

The result of the postal inquiry of 10,000 elderly subjects, with a response rate of $78 \%$, in connection with this study has been reported elsewhere [24]. In brief, it was shown that subjects with underlying chronic disorders had higher vaccination rates $(\mathrm{p}<0.001)$ than those without underlying chronic diseases. A chronic lung disease was noted in $9.2 \%$ among persons vaccinated against influenza and in $9.7 \%$ among persons vaccinated against pneumococcal infection compared with $4.6 \%$ and $5.6 \%$, respectively, for unvaccinated persons. Among the persons who were vaccinated against influenza and pneumococcal infection, $>17 \%$ had a myocardial disease compared with $<12 \%$ among unvaccinated persons. Elderly individuals living in nursing homes or institutions had higher influenza vaccination rates than those living in their own household ( $72 \%$ versus $67 \%$ ).

\section{Discussion}

The present study supports previous findings that influenza and pneumococcal vaccination is effective in reducing hospitalisation and death in elderly subjects [22, 23]. The vaccinated cohort i.e. those receiving one or both vaccines, had an overall lower risk for being hospitalised for the main end-point diagnoses, influenza, pneumonia or IPD, than the nonvaccinated cohort. This effect was consistent through all age groups for pneumonia, but not for influenza and IPD (table 2). It is usually assumed that the effectiveness of both these vaccines decline with increasing age [5, 12]. This was also the case for IPD, where a significant protection was only found in those 65-74 yrs of age. For influenza, however, the opposite was found with protection only in the age groups $>74$ yrs of age. However, it is important to point out that only the incidence of hospital care for influenza was studied, which was very low in the age group 65-74 yrs of age, and that no data on the protection against influenza per se in this age group was obtained.

The large number of individuals receiving only one of the two vaccines, permitted the authors to perform an evaluation of the effectiveness of each vaccine separately. In agreement with earlier retrospective studies [17-20, 25-26], there was a reduction, although not quite significant, of hospitalisation for influenza and pneumonia after influenza vaccination (table 3). 
The effectiveness of pneumococcal vaccination alone in the prevention of pneumonia $(9 \%)$ almost reached statistical significance $(\mathrm{p}<0.06)$. This protective level was comparable with those found during noninfluenza seasons; $12 \%$ during the first study year [23] and $21 \%$ in the present study (table 6), when no additive effect from the influenza vaccine can be expected to occur. Thus, these data strongly indicate that the pneumococcal vaccine alone is $10-20 \%$ effective in the prevention of pneumonia. When also including the influenza season in the analysis, the preventive effect against pneumonia increased from $9 \%(95 \%$ CI $0-18)$ in the group receiving pneumococcal vaccination alone to $29 \%(95 \%$ CI $25-35)$ in those receiving both vaccines (table 3 ). Thus, the two vaccines were clearly additive in the prevention of pneumonia, which corroborate the findings of a recent retrospective cohort study [11]. The surprising tendency that those who received pneumococcal vaccine alone had a lower rate of hospitalisation for influenza (OR $0.70,95 \%$ CI $0.49-1.03$ ), may have been due to that this end-point also included influenza complicated by pneumonia.

The patients who required hospital treatment for influenza or pneumonia despite having received both vaccines had a significantly shorter mean length of stay than those who were not vaccinated. This shorter length of hospital stay could indicate that the vaccinated patients had obtained a partial immunity resulting in a less severe disease. Significantly lower in-hospital mortality was also demonstrated for pneumonia in those who had received both vaccines, compared with the unvaccinated cohort (table 4).

During the influenza season, there were significantly fewer hospital admissions for all end-point diagnoses in the cohort vaccinated with both influenza and pneumococcal vaccine compared with the unvaccinated one, with a reduction of $37 \%$ for influenza, $36 \%$ for pneumonia and $72 \%$ for IPD (table 6 ). The incidences of the investigated diagnoses were generally lower during the noninfluenza season, and a significant lower hospitalisation rate in the vaccinated group was found only for pneumonia.

Compared with the unvaccinated cohort, the reduction of hospital admissions for IPD varied from 53\% (95\% CI 18-73) in the vaccinated cohort (receiving one or both vaccines; table 2) to $73 \%(95 \%$ CI -14-94) for those who received pneumococcal vaccine alone (table 3 ). For the group who were given both vaccines, the reduction was $44 \%(95 \%$ CI -5-70), overall, and $72 \%$ (95\% CI 45-86) during the influenza season. In contrast to the first study year [23], there was no difference during the noninfluenza season between those who had received pneumococcal vaccination and the nonvaccinated cohort concerning the risk for IPD. The latter may have been due to chance, since the incidence of IPD during that time of year was very low and there were only seven cases among those who had received both vaccines and 10 cases in the unvaccinated cohort. The impact on IPD seen in this study corresponds to findings from a pneumococcal and influenza vaccination programme for at-risk indigenous adults in far North Queensland, Australia [27]. Despite a suboptimal vaccine coverage, there was a $75 \%$ reduction of the incidence of IPD, from 111 to 28 cases per 100,000 per year, during the 5-yr programme.

An important question in this study was whether the vaccinated and unvaccinated cohorts were comparable. To exclude the probability that the vaccinated cohort was at a lower risk than the unvaccinated one, a randomised, multivariant study among $\sim 10,000$ of the participants in the vaccination study was performed [24]. It was found that the recipients of the influenza or pneumococcal vaccine were older and more often living in nursing homes and institutions, than those who did not receive the vaccination. In addition, persons in the vaccinated cohort had significantly more chronic lung and heart conditions, than those who were not vaccinated, an observation that also has been described elsewhere [25, 28].

These data indicate that the findings may actually underestimate, rather than overestimate, the beneficial effect of influenza and pneumococcal vaccination. In a recent study by NiCHOL et al. [29], influenza vaccination of elderly patients was associated with a reduction in the risk of hospitalisation for cardiac disease. In contrast to these findings, the present authors found the risk of hospitalisation due to cardiac failure to be the same in the vaccinated and the unvaccinated cohort (data not shown). However, since chronic heart diseases were probably more frequent in the unvaccinated than in the vaccinated cohort [24], the possibility that vaccination had some impact on the risk of hospitalisation for cardiac disease cannot be ruled out in this study population.

The accuracy of using medical record discharge data coded by the ICD for vaccination efficacy studies can be questioned. A recent study showed that while a single ICD code had a low sensitivity, but acceptable specificity, the use of the first five listed codes (not only the first) and groups of codes could increase the sensitivity without a substantial change in specificity, positive or negative predictive values [30].

To conclude, these findings confirm that vaccination of elderly individuals with influenza and pneumococcal vaccines is effective in preventing hospitalisation and mortality caused by influenza, pneumonia and invasive pneumococcal disease. Although the influenza and pneumococcal vaccines seem to be effective when used separately, the results of the present study also indicate an additive benefit of immunising elderly persons with both vaccines.

\section{References}

1. Nichol KL. The additive benefit of influenza and pneumococcal vaccinations during influenza seasons among elderly persons with chronic lung disease. Vaccine 1999; 17: S91S93.

2. Centers for Disease Control and Prevention. Influenza and pneumococcal vaccination levels among adults aged $>65$ years, United States, 1997. MMWR 1998; 47: 797-802.

3. Govaert ME, Thijs CTMCN, Masurel N, Sprenger MJW, Dinant GJ, Knottnerus JA. The efficacy of influenza vaccination in elderly individuals. JAMA 1994; 272: 16611665.

4. Fedson DS, Hirota Y, Shin HK, et al. Influenza vaccination in 22 developed countries: an update to 1995. Vaccine 1997; 15: $1506-1511$.

5. Centers for Disease Control and Prevention. Prevention and control of influenza: Recommendations of the Advisory Committee on Immunization Practices. MMWR 1997; 46: 125.

6. American College of Physicians. Guide for Adult Immunization. 3rd Edn. Philadelphia, American College of Physicians, 1994.

7. Simberkoff MS, Cross AP, Al-Ibrahim M, et al. Efficacy of pneumococcal vaccine in high-risk patients. $N$ Engl J Med 1986; 315: 1318-1327.

8. Örtquist $\AA$, Hedlund J, Burman L-Å, et al. Randomised trial of 23-valent pneumococcal capsular polysaccharide vaccine in prevention of pneumonia in middle-aged and elderly people. Lancet 1998; 351: 399-403.

9. Koivula I, Sten M, Leinonen M, Mäkelä PH. Clinical efficacy of pneumococcal vaccine in the elderly: a randomized, single-blind, population-based trial. Am J Med 1997; 103: $281-290$.

10. Gaillat J, Zmirou D, Mallaret MR, et al. Clinical trial of pneumococcal vaccine among institutionalised elderly [Essai 
clinique du vaccin anti-pneumococcique chez des personnes] Rev Epidémiol Santé Publique 1985; 33: 437-444.

11. Nicol KL, Baken L, Wuorenma J, Nelson A. The health and economic benefits associated with pneumococcal vaccination of elderly persons with chronic lung disease. Arch Intern Med 1999; 159: 2437-2442.

12. Shapiro ED, Berg AT, Austrian R, et al. The protective efficacy of polyvalent pneumococcal polysaccharide vaccine. $N$ Engl J Med 1991; 325: 1453-1460.

13. Farr BM, Johnston BL, Cobb DK, et al. Preventing pneumococcal bacteremia in patients at risk. Results of a matched case-control study. Arch Intern Med 1995; 155: 2336-2340.

14. Sims RV, Steinmann WC, McConville JH, King LR, Zwick WC, Schwartz JS. The clinical effectiveness of pneumococcal vaccine in the elderly. Ann Intern Med 1988; 108: 653-657.

15. Butler JC, Breiman RF, Campbell JF, Lipman HB, Broome CV, Facklam RR. Pneumococcal polysaccharide vaccine efficacy. An evaluation of current recommendations. JAMA 1993; 270: 1826-1831.

16. Barker WH, Mullooly JP. Pneumonia and influenza deaths during epidemics: implications for prevention. Arch Intern Med 1982; 142: 85-89.

17. Foster DA, Talsma A, Furumoto-Dawson A, et al. Influenza vaccine effectiveness in preventing hospitalization for pneumonia in the elderly. Am J Epidemiol 1992; 136: 296-307.

18. Fedson DS, Wajda A, Nicol P, Hammond GW, Kaiser DL, Roos LL. Clinical effectiveness of influenza vaccination in Manitoba. JAMA 1993; 270: 1956-1961.

19. Mullooly JP, Bennett MD, Hornbrook MC, et al. Influenza vaccination programs for elderly persons: cost-effectiveness in a health maintenance organization. Ann Intern Med 1994; 121: 947-952.

20. Nichol KL, Margolis KL, Wuorenma J, von Sternberg T. The efficacy and cost effectiveness of vaccination against influenza among elderly persons living in the community. N Engl J Med 1994; 331: 778-784.
21. Ahmed AH, Nicholson KG, Nguyen-van Tam JS, Pearson JCG. Effectiveness of influenza vaccine in reducing hospital admissions during the 1989-90 epidemic. Epidemiol Infect 1997; 118: 27-33.

22. Christenson B, Lundbergh P, Hedlund J, Örtqvist Å. Effects of a large-scale intervention with influenza and 23 -valent pneumococcal vaccines in adults 65 years and older: a prospective study. Lancet 2001; 357: 1008-1011.

23. Hedlund J, Christenson B, Lundbergh P, Örtqvist Å. Effects of a large-scale intervention with influenza and 23-valent pneumococcal vaccines in elderly people: A one-year followup. Vaccine 2003; 21: 3906-3911.

24. Christenson B, Lundbergh P. Comparison between cohorts vaccinated and unvaccinated against influenza and pneumococcal infection. Epidemiol Infect 2002; 129: 515-524.

25. Nichol KL, Wuorenma J, von Sternberg T. Benefits of influenza vaccination for low-, intermediate-, and high-risk senior citizens. Arch Intern Med 1998; 158: 1769-1776.

26. Gross PA, Hermogenes AW, Sacks HS, Lau J, Lewandowski RA. The efficacy of influenza vaccine in elderly persons. A meta-analysis and review of the literature. Ann Intern Med 1995; 123: 518-527.

27. Hanna JN, Young DM, Brookes DL, Dostie GB, Murphy DM. The initial coverage and impact of the pneumococcal and influenza vaccination program for atrisk indigenous adults in Far North Queensland. Aust NZ J Public Health 2001; 25: 543-546.

28. Gillick MR, Ditzon B. Influenza vaccination: are we doing better than we think? Arch Intern Med 1991; 151: 1742-1744.

29. Nichol KL, Nordin J, Mullooly J, Lask R, Fillbrandt K, Iwane $\mathrm{M}$. Influenza vaccination and reduction in hospitalizations for cardiac disease and stroke among the elderly. $N$ Engl J Med 2003; 348: 1322-1332.

30. Guevara RE, Butler JC, Marston BJ, Plouffe JF, File TM, Breiman RF. Accuracy of ICD-9-CM codes in detecting community-acquired pneumococcal pneumonia for incidence and vaccine efficacy studies. Am J Epidemiol 1999; 149: 282289. 